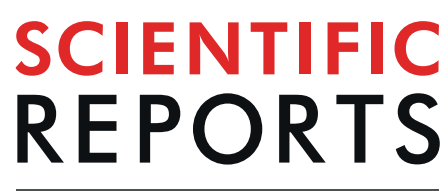

natureresearch

\title{
OPEN Characterization of the Long Terminal Repeat of the Endogenous Retrovirus-derived microRNAs in the Olive Flounder
}

Received: 31 May 2018
Accepted: 12 September 2019

Published online: 30 September 2019

\begin{abstract}
Hee-Eun Lee ${ }^{1,2}$, Ara Jo ${ }^{1,2}$, Jennifer $\mathrm{Im}^{1,2}$, Hee-Jae Cha ${ }^{3}{ }^{3}$, Woo-Jin Kim ${ }^{4}$, Hyun Hee Kim ${ }^{5,6}$, Dong-Soo Kim ${ }^{7}$, Won $\mathrm{Kim}^{8}$, Tae-Jin Yang ${ }^{9}{ }^{9}$ \& Heui-Soo Kim ${ }^{2,10}$

Endogenous retroviruses (ERVs) have been identified at different copy numbers in various organisms. The long terminal repeat (LTR) element of an ERV has the capacity to exert regulatory influence as both a promoter and enhancer of cellular genes. Here, we describe olive flounder (OF)-ERV9, derived from chromosome 9 of the olive flounder. OF-ERV9-LTR provide binding sites for various transcription factors and showed enhancer activity. The OF-ERV9-LTR demonstrates high sequence similarity with the $3^{\prime}$ untranslated region (UTR) of various genes that also contain seed sequences (TGTTTTG) that bind the LTR-derived microRNA(miRNA), OF-miRNA-307. Additionally, OF-miRNA-307 collaborates with transcription factors located in OF-ERV9-LTR to regulate gene expression. Taken together, our data facilitates a greater understanding of the molecular function of OF-ERV families and suggests that OFmiRNA-307 may act as a super-enhancer miRNA regulating gene activity.
\end{abstract}

Paralichthys olivaceus, known as olive flounder (OF) is an economically important marine flatfish which is extensively cultured in Korea, China and Japan. Due to their high economic value, there are several selective breeding programs in place, such as those involving sex manipulation, owing to differences in growth speed and size between male and female olive flounders ${ }^{1-3}$. However, due to farming conditions, olive flounders are prone to fatal infections or diseases caused by various bacteria, viruses and parasites ${ }^{4-7}$. The olive flounder RNA sequencing program has provided important information regarding known alternative splicing patterns and gene duplication events within the olive flounder ${ }^{8}$. However, there are few studies which characterize olive flounder from a molecular biology approach.

Endogenous retroviruses (ERVs) are one of the transposable elements (TE) which are inherited as stable genomic components throughout the evolution of a species. Depending on the species, copy number and chromosomal distribution may vary ${ }^{9}$. ERVs mediate structural variation and genomic instability based on their copy number and reverse transcriptase activity ${ }^{10}$. ERV elements are highly defective, containing large deletions, stop codons, and frameshifts in their open reading frames (ORFs). Moreover, structural genes from some ERV families are preferentially expressed in various tissues and cancer cell lines ${ }^{11,12}$. Multiple-copy of ERV families scattered throughout the genome have been reported to regulate the expression of neighbouring genes ${ }^{13-15}$. Long terminal repeat elements (LTR) recruit transcription factors and thus can enhance the transcription of host cell genes ${ }^{11,15}$. 
(A)

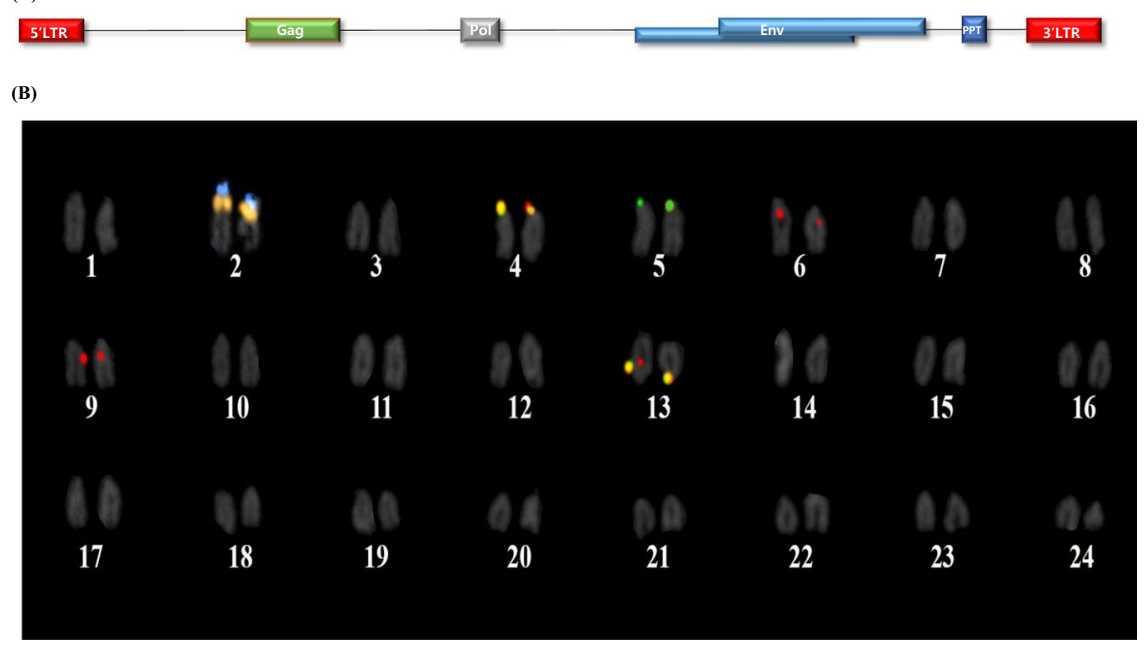

Figure 1. (A) The schematic structure of OF-ERV9, created using RetroTector. In addition to a 5' and 3LTR, this sequence includes genes encoding the gag, pol, env and ppt proteins. (B) Karyogram of a female olive flounder showing $5 \mathrm{~S}$ (blue) and 45S (orange) rDNA, OF-ERV5-LTR (green), and OF-ERV9-LTR (red). Colocalization of two LTR elements is indicated in yellow.

LTR elements may contain intrinsic enhancer activity; however, base substitutions, transcription factor binding sites (TFBS), bi-directional transcription start sites (TSS), and open chromatin may increase the enhancer activity of LTR elements ${ }^{16}$. Tissue-specific promoter and enhancer activity of human endogenous retrovirus (HERV) -K LTR has been shown in several human and CHO cell lines ${ }^{17}$. Furthermore, studies have shown that species-specific ERV enhancer activity is generally restricted to hypomethylated tissues, suggesting that ERV families are an important mediator in evolutionary regulation ${ }^{18}$. In the case of olive flounder, chromosome 5-derived OF-ERV5-LTR has shown promoter activity in HepG2 and HINAE cells which prompted us to investigate the role of OF-ERV9-LTR in this study ${ }^{19}$.

MicroRNAs (miRNAs) are small noncoding RNA molecules made up of 19 to 22 nucleotides (nt), that play important roles in gene regulation ${ }^{20-22}$. Primary miRNAs (pri-miRNAs) are a long double-stranded RNA (dsRNA) transcript with a hairpin structure, which is identified by the nuclear protein DiGeorge Syndrome Critical Region 8 (DGCR8). The RNA-binding protein DGCR8 together with the RNase III enzyme Drosha recognize and cleave the hairpin RNA, thereby turning pri-miRNA into pre-miRNA. The formed pre-miRNA also adopts a hairpin structure which is processed into mature miRNAs by Dicer. Individual miRNAs bind to $3^{\prime}$ untranslated region (UTR) of target gene through a critical region called the "seed region", in order to regulate gene expression by either translational repression or mRNA degradation ${ }^{20}$. Previously, TE-derived human miRNA genes were discovered, and it was shown that several miRNA genes share their sequences with TEs ${ }^{23,24}$.

In this study, the genomic structure, chromosomal location and enhancer activity of OF-ERV9-LTR have been analysed. The enhancer activity of OF-ERV9-LTR was controlled by TFBSs. In addition, a novel OF-ERV9-LTR-derived miRNA was discovered. The relative expression and functional studies of the OF-ERV9-LTR-derived microRNA-307 (OF-miR-307) suggest that OF-ERV-LTR elements may contribute to several biological functions in olive flounder.

\section{Results}

Structure and chromosomal location of OF-ERVs. The schematic structure of OF-ERV9 shows that each terminal contains an LTR, with the $g a g$, pol, env, and ppt genes placed between them (Fig. 1A). The reference $5 \mathrm{~S}$ and $45 \mathrm{~S}$ rDNA repeats were distinctly observed in the metaphase chromosome spread of the olive flounder, with one pair of each $5 \mathrm{~S}$ and $45 \mathrm{~S}$ rDNA located side by side on the short arms, near the centromeric region of the sub-telocentric chromosome 2. Three pairs of OF-ERV5-LTR signals were observed on the short arm of chromosome 4 and 5 and on the long arm of chromosome 13 (Fig. 1B). Meanwhile, four pairs of OF-ERV9-LTR signals were observed, which were localized on chromosomes 4, 6, 9 and 13. The signals on chromosome 6 and 9 were detected in the pericentromeric region. In the case of the two homologous chromosome pairs on chromosomes 4 and 13, the yellow signal suggested that OF-ERV5-LTR and OF-ERV9-LTR were located very close to each other, due to the overlap of the green and red signals.

Sequence analysis of OF-ERV9-LTR and enhancer activity analysis in SW620 cells. The TFBSs of OF-ERV9-LTR constructs were analysed by using MATCH in TRANSFAC v8.0. (Suppl Fig. 1). The TRANSFAC program was used to predict TFBSs and those that had a threshold value higher than 0.95 for both core match and matrix match were selected and labelled. Constructs containing the OF-ERV9-LTR region and various permutations were cloned into the pGL-4.23 vector (Promega) and checked for enhancer activity (Fig. 2). The plasmid containing the OF-ERV9-LTR region showed high enhancer activity. This enhancer activity was downregulated when the construct excluded the binding site for the transcription factor (TF) Sox-5. In contrast, the activity 


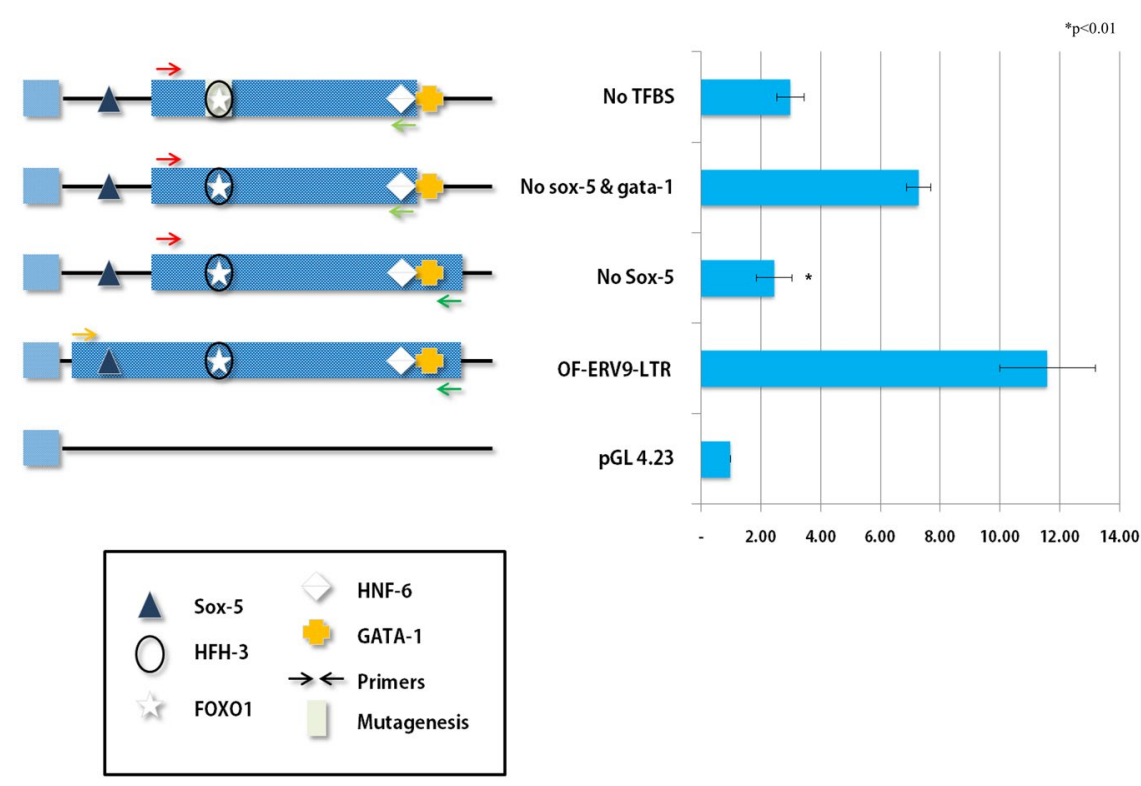

Figure 2. The enhancer activity analysis of OF-ERV9-LTR. Each OF-ERV9-LTR region was cloned into an enhancer vector, as indicated by the schematic structures, and assayed in a cell culture. The structure on the left side describes the vector as well as which transcription factors were cloned. Each arrow represents differently designed primers, described in detail in the Materials and Methods section of the main text. The graph shows the enhancer activity of each cloned plasmid. The data presented represent the mean \pm standard error (Student's t-test vs. control; * $\mathrm{p}<0.01)$.

increased slightly when Sox-5, GATA-1 and HNF-6 binding sites were excluded. Lastly, point mutations were induced in the binding sites of FOXO1 and HFH-3, enabling the exclusion of all the identified and selected TFBSs. In this instance, enhancer activity was further downregulated.

Identification and phylogenetic relationship of OF-ERV9-LTR sequences. OF-ERV9-LTR sequences were analysed using the NCBI database to identify genes with similar sequences. As a result, the olive flounder genes $m k \ln 1$, slc37a3, LOC109642524, LOC109644478, LOC109626170, and LOC109636349 were matched with the OF-ERV9-LTR sequence. The identified conserved region is highlighted by a red box in Fig. 3A. A new miRNA, OF-miRNA-307 (LC333100), was identified in the conserved region of the 3'-UTR of $m k \ln 1$ and OF-ERV9-LTR (Fig. 4A). The red box in Fig. 4A indicates the seed region of miRNA-307, which binds to the 3'-UTR of both $m k \ln 1$ and OF-ERV9-LTR. This region is also indicated in Fig. 3A by a red line. The sequence alignment of OF-ERV9-LTR, $m k \ln 1$, slc37a3, LOC109642524, LOC109644478, LOC109626170 and LOC109636349 was visualized by using WebLogo ${ }^{25,26}$ (Fig. 3D), where the blue box indicates conserved regions. The sequences of all six genes and OF-ERV9-LTR were then used to create a phylogeny tree (Fig. 3B). Mkln 1 shows a close relation to slc37a3, followed by LOC10964524. On the other hand, OF-ERV9-LTR has greater homology to LOC109644478. Additional evidence for this similarity was provided by the dottup program, where the long, even central line indicates high similarity between the 3'-UTR of $m k \ln 1$ and OF-ERV9-LTR (Fig. 3C).

RNA hybrid structure of miRNA and its target genes. The conserved sequences of OF-ERV9-LTR, which generated OF-miRNA-307, were hybridized with target genes as shown in Supplementary Fig. 2, and the minimum free energy for hybridization was calculated. The RNAhybrid energy scale for OF-miRNA-307 and LOC109644478 was $-17.2 \mathrm{kcal} / \mathrm{mol}$ (Suppl. Fig. 2A), for $m k \ln 1$ it was $-20.2 \mathrm{kcal} / \mathrm{mol}$ (Suppl Fig. 2B), for LOC109636349 it was $-34.5 \mathrm{kcal} / \mathrm{mol}$ (Suppl. Fig. 2C) and for slc37a3 it was $-17.8 \mathrm{kcal} / \mathrm{mol}$ (Suppl. Fig. 2D). The lowest energy scales denote a strong interaction between the two molecules. As $m k \ln 1$ showed the second lowest energy scale, $m k \ln 1$ was selected for further study. Despite the fact that LOC109636349 has the lowest energy scale, it was not selected since it is uncharacterized and is annotated as a noncoding RNA, while $m k \ln 1$ is a protein-coding gene.

Analysis of OF-miRNA-307 and related miRNAs. The sequence of OF-miRNA-307 was analysed by using the miR-base website in order to examine if there are any related miRNAs. As a result, six related miRNAs with conserved regions were identified (Suppl. Fig. 3). Four of these miRNAs were of human origin, belonging to the miRNA-642 family. The other miRNAs belonged to Pan troglodytes and Gossypium. Of all six analogous miRNAs, hsa-miRNA-642a-5p-RC and ptr-miRNA-642-RC had the highest degree of homology with OF-miRNA-307. As hsa-miRNA-642a-5p-RC was highly conserved, it was used in order to analyse biological processes, cellular components and molecular functions in Gene Ontology ${ }^{27}$ (Suppl. Fig. 4). Metabolic and 
(A) LoC109642524

OF-ERV9-LTR

LOC109644478

mkln1

LOC109626170

LOC109636349

slc37a3

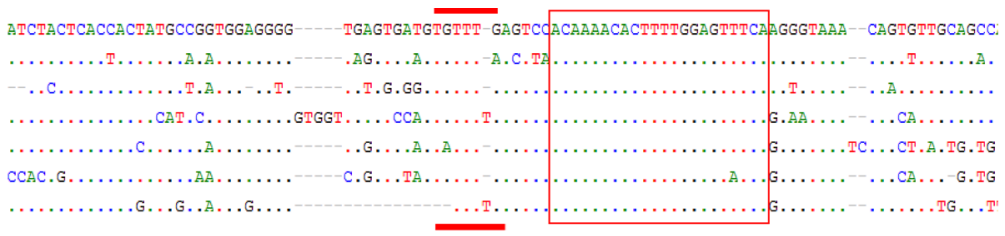

(B)

(C)
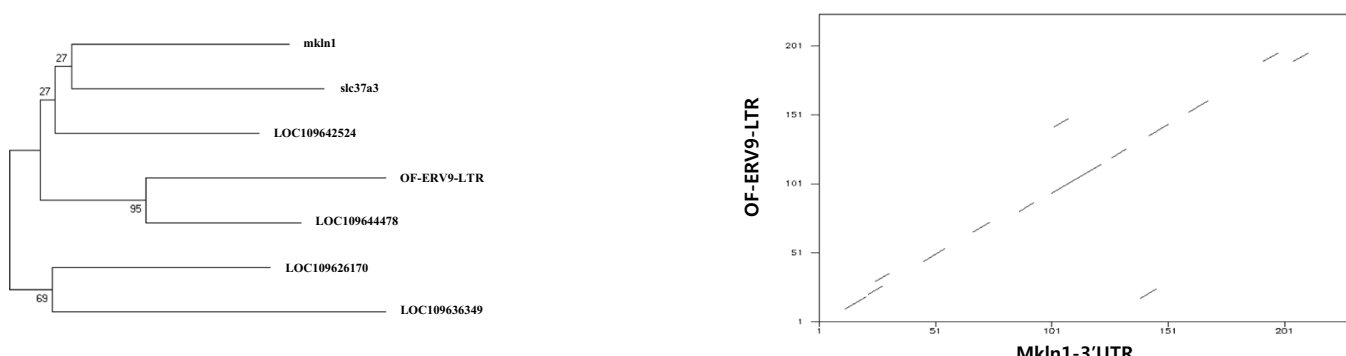

$\longmapsto 0.050$

Mkln1-3'UTR

(D)

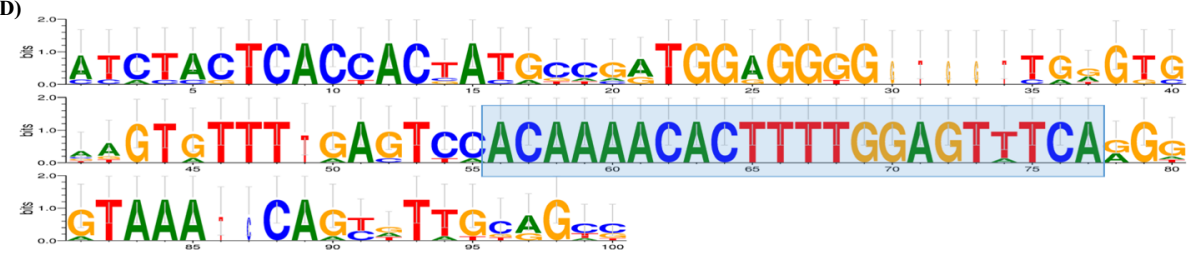

Figure 3. (A) OF-ERV9-LTR was analysed in comparison to related target gene sequences. The red line denotes the seed region of OF-miRNA-307 and the red box indicates the conserved region of all genes. (B) Phylogenetic tree comparing OF-ERV9-LTR and related genes. Scale bar $=0.05$. (C) Dot plot comparison of the OF-ERV9-

LTR and $m k \ln 13$ '-UTR sequences. The $\mathrm{x}$ axis represents the $3^{\prime}$-UTR of the $m k \ln 1$ gene and the y axis represents the LTR of OF-ERV9. (D) Sequence alignment of OF-ERV9-LTR and its related genes using the Web Logo software. The blue box indicates the conserved region.
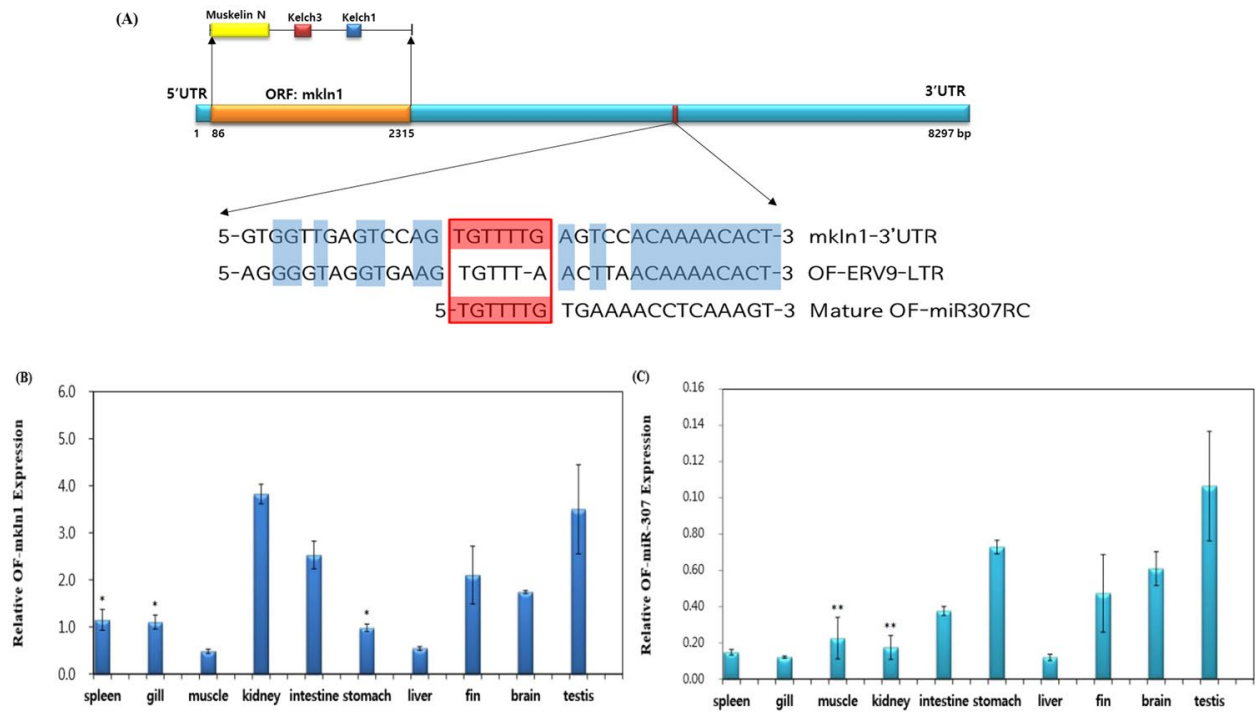

Figure 4. (A) Schematic structure of the $m k \ln 1$ gene. The Muskelin N, Kelch3 and Kelch1 proteins are contained in the ORF region of the $m k \ln 1$ gene. The sequences coloured in blue are conserved regions and the red box is the seed region of the OF-miRNA-307. (B) Relative quantitative PCR expression patterns for $m k \ln 1$ in various tissues. Amongst all the olive flounder tissues tested, the kidney, intestine, fin and testis showed the highest expression levels of $m k \ln 1$. (C) Relative quantitative PCR expression patterns for OF-miRNA-307. Of all the tissues tested, the lowest expression of OF-miRNA-307 was found in the spleen, gills, kidneys and liver. The data presented represent the mean \pm standard error (Student's t-test vs. control; * $\mathrm{p}>0.15, * * \mathrm{p}>0.02$ ). 


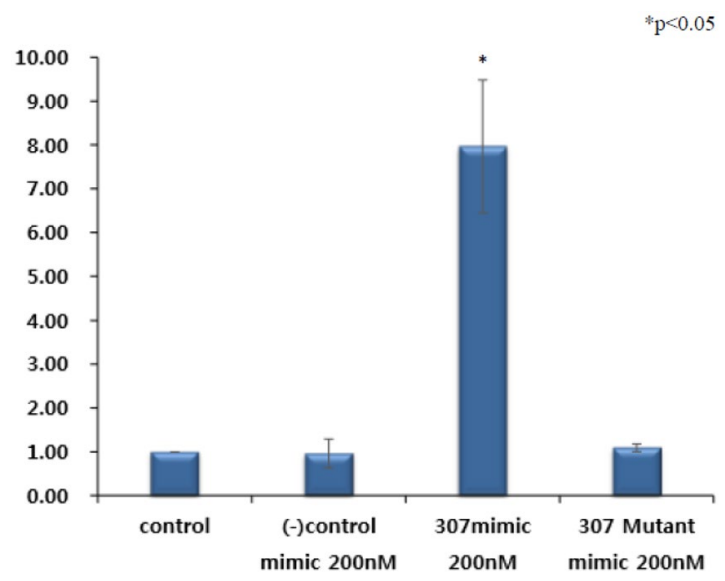

Figure 5. (A) Co-transfection of the $m k \ln 13^{\prime}$-UTR and OF-miRNA-307 mimic. In contrast to the negative control and OF-miRNA-307 mimic mutant, co-transfection of the $m k \ln 13^{\prime}$-UTR with the OF-miRNA-307 mimic induced a dramatically increase in expression level. The data presented represent the mean \pm standard error (Student's t-test vs. control; * $\mathrm{p}<0.05$ ).

cellular processes were the most abundant amongst the analysed results, with cell part, catalytic activity and binding activity also being strongly represented.

Relative expression analyses of $m k \ln 1$ and OF-miRNA-307. The expression of $m k \ln 1$ and OF-miRNA-307 in various OF tissues was evaluated by qPCR (Fig. 4B,C). The relative expression of $m k \ln 1$ in OF kidneys, intestine, fin, and testis tissues was higher than in other OF tissues (Fig. 4B). On the other hand, the spleen, gills, kidneys, and liver showed low expression of OF-miRNA-307 (Fig. 4C).

Co-transfection of the $m k \ln 13^{\prime}$-UTR and OF-miRNA-307 mimics. We hypothesized that the co-transfection of $m k \ln 13^{\prime}$-UTR and OF-miRNA-307 mimics in HT-29 cells would result in a lower or similar expression of the OF-miRNA-307 mimic compared to controls and that the OF-miRNA-307 mutant mimic would be overexpressed. However, we found that the OF-miRNA-307 mimic was highly expressed, at a level much greater than the mutant or any of the controls (Fig. 5).

\section{Discussion}

Previous studies regarding olive flounder focused on preventing infections and improving farming conditions, providing valuable outcomes. However, the molecular biological characteristics of olive flounders are still poorly understood. Therefore, the present study aimed to characterize the OF-ERV9-LTR through various bioinformatics tools, in order to provide fundamental information on this subject. As a result, a newly reported miRNA has been identified and described.

ERVs contain enormous deletions, as well as stop codons and frameshifts in their ORFs, and copy number and chromosomal distributions vary between species. TEs insert themselves into any part of the genome, thereby creating novel sequences in the genome which leads to the generation of new genes ${ }^{9,10,13-15}$. The ERV9 found in olive flounder has well conserved LTRs, including gag, pol, env and $p p t$ sequences. A previous study has shown that OF-ERV5 also has well-conserved sequences among olive flounder and confirmed the promoter activity of OF-ERV5, 9 and 10 in both HINAE and HepG2 cell lines ${ }^{19}$. The present study is mainly focused on the OF-ERV9-LTR, another highly conserved and widely spread OF-ERV in olive flounder and supports the idea that both TEs truly are "jumping genes". Alternative promoter and enhancer activity are an additional function of TE, which can act as both a promoter and an enhancer by inserting itself into specific genes ${ }^{9,28,29}$. The present study suggests that OF-ERV9-LTR mainly has enhancer activity, despite having a weak promoter activity ${ }^{19}$. Moreover, TEs provide binding sites for TFs, thereby greatly influencing gene regulation ${ }^{18,30-32}$. It has been previously demonstrated that the tandem repeat region of the LTR12C element is critical for its promoter activity, with or without $\mathrm{TFs}^{33}$. Therefore, we decided to eliminate TFBSs nearby OF-ERV9-LTR, based on previous results which suggested that enhancer activity is partially controlled by transcription factors. To examine primary TFs that have enhancer function along with OF-ERV9-LTR, a deletion variant of each TF was designed. Our results suggest that the transcription factor SOX-5 had the strongest enhancer activity when compared with other analysed transcription factors, namely GATA-1, HFH-3, FOXO1 and HNF-6.

The transcription factor SOX-5 is involved in embryonic development, cell fate and differentiation ${ }^{34,35}$. Previous studies have revealed that Sox-5 is an enhancer, as well as a super-enhancer (SE) in a variety of biological processes ${ }^{36-39}$. The transcription factor SOX-5 in OF-ERV9-LTR is located in the region between $166 \mathrm{bp}$ and $170 \mathrm{bp}$, while the OF-miRNA-307 was located in the region between $103 \mathrm{bp}$ and $108 \mathrm{bp}$. The close positioning of both Sox-5 and OF-miRNA-307 within the OF-ERV9-LTR can lead to the generation of enhancer RNA (eRNA). One study indicated that eRNA plays a role in regulating transcription and that it may also act as an activator rather than a repressor of the target promoter region ${ }^{40}$. 
Functional Gene

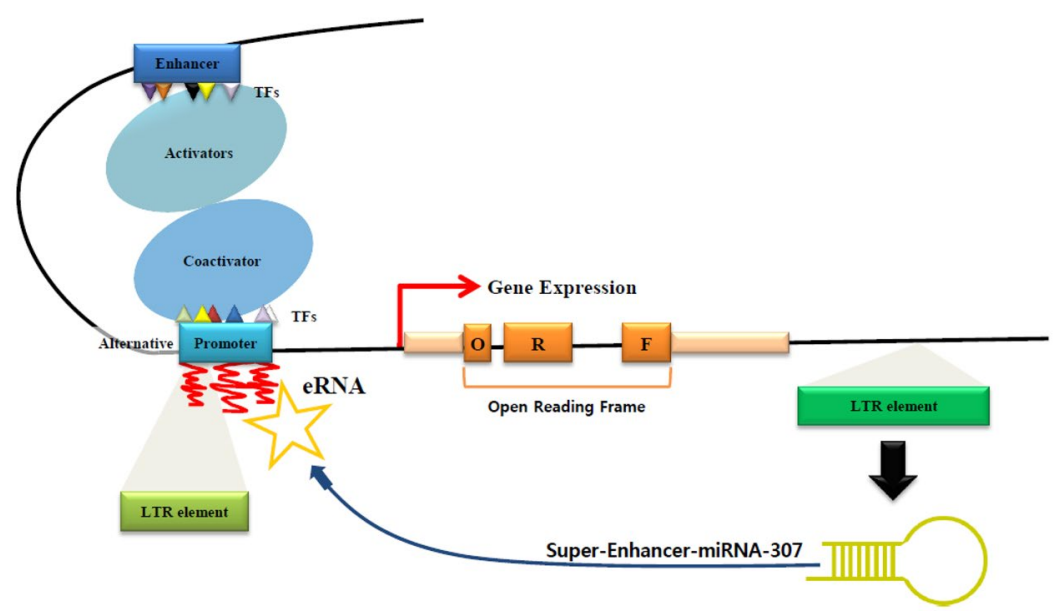

Figure 6. Schematic indicating the predicted roles of OF-miRNA-307. It is possible that OF-miRNA-307 functions as an SE-miRNA and that this SE-miRNA activates the generation of eRNA, resulting in a dramatic increase in the expression of the target gene.

miRNAs derived from TEs have been identified in several studies, which showed that various types of TEs, such as long interspersed elements (LINE), short interspersed elements (SINE), LTRs and DNA transposons, can give rise to several miRNA families ${ }^{23,24,41,42}$. Furthermore, these studies suggest that TEs may have affected mammalian evolution by inducing the creation of new miRNAs. TE-derived miRNAs not only bind to the $3^{\prime}$-UTR of target genes but can also bind to their $5^{\prime}$-UTR ${ }^{23}$. In the case of OF-ERV9-LTR, it can generate both OF-miRNA-307 and the $3^{\prime}$-UTR of the $m k \ln 1$ gene (Fig. 4). The OF-ERV9-LTR inserted itself into the olive flounder genome thereby creating the OF-miRNA-307. A few studies have shown that miRNAs that act as an enhancer when binding to the $3^{\prime}$-UTR region of its target gene are called super-enhancer-miRNAs (SE-miRNA) ${ }^{43,44}$. We hypothesize that the OF-miRNA-307 may function as a SE-miRNA by cooperating with the super-enhancer TF SOX-5 (Fig. 6). The OF-ERV9-LTR-derived miRNA, OF-miRNA-307, is located in the region neighbouring the enhancer or super-enhancer TF called SOX-5 and can either act as an enhancer or augment the already existing enhancer function of SOX-5. Subsequently, either the enhancer or SOX-5 recruit activators and co-activators to the promoter region of the functional gene. Meanwhile, SE-OF-miRNA-307 collaborates with either the gene promoter or the alternative promoter generated by the LTR element. As such, with the cooperation of the super-enhancer TF SOX-5, OF-miRNA-307 may function as a SE-miRNA in the $m k l n 1$ gene.

We suggest that OF-miRNA-307 may function as a SE-miRNA, through a collaboration with the TF SOX5. Analysing and determining the enhancer function of OF-miRNA-307 requires further research. However, some studies have already revealed and predicted that enhancer miRNA may play several crucial roles. Our findings illustrate another example of enhancer miRNA activity. Hence, we presume that OF-ERV9-LTR derived OF-miRNA-307 may function as a SE-miRNA, thereby regulating and enhancing gene transcription.

\section{Materials and Methods}

Ethical statement. All experiments in this study were carried out in accordance with the guidelines and regulation approved by Pusan National University-Institutional Animal Care and Use Committee (PNU-IACUC).

Isolation of genomic DNA and RNA from olive flounder. The genomic DNA (gDNA) of olive flounder was extracted from blood by using the DNeasy Blood \& Tissue Kit (Qiagen, Valencia, CA) according to the manufacturer's protocol. Extracted gDNA samples were then used for PCR amplification. Total RNA was extracted from 11 healthy olive flounder tissue samples using TRIzol (Invitrogen, Carlsbad, CA) with RNase-Free DNase (New England Biolabs, Beverly, MA) according to the manufacturer's protocol. The quantity and quality of each gDNA and RNA sample were determined using an ND-1000 UV-Vis spectrophotometer (NanoDrop, Wilmington, DE). The PrimeScript ${ }^{\mathrm{TM}}$ RT reagent Kit with gDNA Eraser (TaKaRa, Japan) was used for cDNA synthesis and gDNA removal from $0.5 \mu \mathrm{g}$ of each total RNA sample.

Computational data analysis of the LTR-derived OF-miR-307. OF-ERV9-LTR-derived sequences were retrieved from the NCBI database (https://www.ncbi.nlm.nih.gov/). The structure of OF-ERV9 was drawn based on the program RetroTector ${ }^{45}$. The OF-ERV9 5LTR sequence was analysed with BLAST to discover similar families. Following that, sequence alignments were analysed using BioEdit ${ }^{46}$. All aligned sequences were then used to generate a phylogeny tree using MEGA $7^{47}$. The WebLogo program was used to display the conserved sequences in a graphic view ${ }^{25,26}$. The Dottup program (http://www.bioinformatics.nl/cgi-bin/emboss/dottup) was used to check for analogous regions between the 5'LTR of OF-ERV9 and the 3' UTR region of $m k \ln 1$. Prediction of LTR-derived OF-miRNA-307 secondary structures was performed by employing the RNAfold WebServer (http://rna.tbi.univie.ac.at/cgi-bin/RNAWebSuite/RNAfold.cgi), considering both seed region pairing and 
minimum free energy. In order to predict OF-miRNA-307 targets, OF-miRNA-307 was hybridized with target genes through RNAhybrid in the web program BiBiServ (https://bibiserv.cebitec.uni-bielefeld.de/) ${ }^{48}$. The gene ontology program was used to analyse molecular function, cellular components and biological processes of the miRNA-307 analogous miRNA, hsa-miRNA-642a-5p-RC (http://www.geneontology.org/)27.

Fluorescence in situ hybridization (FISH) of OF-ERVs in olive flounder. A kidney cell suspension was prepared according to the protocol established by Kim $(1982)^{49}$. Briefly, cells were spread onto pre-cleaned slides in a humid chamber and air-dried. The slides were subsequently fixed with $2 \%$ formaldehyde, dehydrated in a graded ethanol series $(70 \%, 90 \%$, and 100\%) and air-dried. The FISH procedure was done as described by Waminal $(2012)^{50}$ with small modifications. The typically conserved eukaryote FISH signals of $5 \mathrm{~S}$ and $45 \mathrm{~S}$ rDNA $^{51}$ were used as reference signals and labelled by PCR with digoxygenin-11-dUTP and biotin-16-dUTP, respectively. Plasmid DNAs, OF-ERV5-LTR and OF-ERV9-LTR, derived from olive flounder, were denatured by boiling for $5 \mathrm{~min}$ and then labelled with digoxygenin-11-dUTP and biotin-16-dUTP through nick-translation (Roche, Germany). Chromosome spread slides were treated with $100 \mu \mathrm{g} / \mathrm{mL}$ RNase A in $2 \times$ SSC for one hour at $37^{\circ} \mathrm{C}$. Slides were incubated in both $0.01 \mathrm{~N} \mathrm{HCI}$ for $2 \mathrm{~min}$ at room temperature and $0.005 \%$ diluted pepsin in $0.01 \mathrm{~N} \mathrm{HCI}$ for $10 \mathrm{~min}$ at $37^{\circ} \mathrm{C}$ and washed with $2 \times$ SSC twice. Slides were post-fixed in $4 \%$ paraformaldehyde solution for 10 minutes, washed in $2 \times$ SSC, dehydrated in a graded ethanol series $(70 \%, 90 \%$, and $100 \%)$ and then air-dried.

Digoxigenin-labelled OF-ERV5-LTR and 5S rDNA were detected using monoclonal anti-digoxigenin-fluorescein isothiocyanate (FITC) conjugates (Sigma, USA). Biotinylated OF-ERV9-LTR and 45S rDNA probes were detected using $\mathrm{Cy}^{\mathrm{TM}} 3$-streptavidin conjugates (Zymed, USA). Incubated slides were washed in detection buffer at $37^{\circ} \mathrm{C}$ and subjected to dehydration in an ethanol series. Slides were air-dried and counterstained with $1 \mu \mathrm{g} / \mathrm{mL}$ 4'-6-diamidino-2-phenylindole (DAPI) (Roche, Germany) in a Vectashield (Vector Lab, Inc., Burlingame, CA) solution. Then, chromosomes were observed under an Olympus BX53 fluorescence microscope equipped with a Leica DFC365 FS CCD camera, using an oil-immersion lens ( $\times 100$ magnification). Captured images were processed using

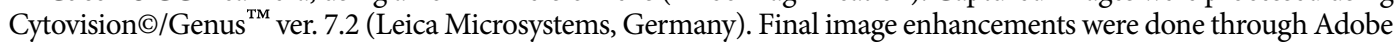
Photoshop CC (Adobe Systems, San Jose, CA).

Cloning of the OF-ERV9-LTR gene. In order to perform gene cloning, primers were designed to amplify

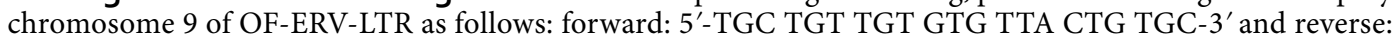
5'-CAT GAC AAC AAA GGA TGC TCA-3'. Primers for variants of Sox-5 containing deletions designed as follows: 5'-ACT GAT CGA TTT TTC AAA CG-3' as the forward primer and 5'-GCA ATG CTA GCA GAA GAT TA- $3^{\prime}$ as the reverse primer. The variants of GATA-1, HNF6, HFH-2 and FOXO1 that contained deletions shared the same forward primer with the Sox-5 deleted variant and the reverse primer was designed as $5^{\prime}$-TGA TTT TAA CAT GCA ACC TG-3'. Genomic PCR primers were designed using Primer $3^{52,53}$.

PCR was carried out in a total reaction volume of $25 \mu \mathrm{L}$, containing $2.5 \mu \mathrm{L}$ of $10 \mathrm{X}$ PCR buffer, $3 \mu \mathrm{L}$ of each dNTP (stock concentration, $2.5 \mu \mathrm{M}$ ), $0.1 \mu \mathrm{L}$ of Ex Taq polymerase (TaKaRa, Japan), $16.4 \mu \mathrm{L}$ of ddH $\mathrm{H}_{2} \mathrm{O}, 1 \mu \mathrm{L}$ of each primer and $1 \mu \mathrm{L}$ of gDNA. After an initial denaturation step at $95^{\circ} \mathrm{C}$ for $5 \mathrm{~min}$, the products were amplified for 30 cycles of $95^{\circ} \mathrm{C}$ for $30 \mathrm{sec}, 55^{\circ} \mathrm{C}$ for $30 \mathrm{sec}$ and $72^{\circ} \mathrm{C}$ for $90 \mathrm{sec}$, with a final elongation step at $72^{\circ} \mathrm{C}$ for $5 \mathrm{~min}$. The PCR products were separated on a $1.5 \%$ agarose gel, purified with the Labo Pass Kit (Cosmogenetech, Korea), and cloned into the pGL-4.23 vector (Promega, USA). The cloned plasmid products were then isolated with the Hybrid-Q Plasmid mini Kit (GeneAll, Korea) and sequenced by Cosmogenetech.

Quick change mutagenesis. Quick change mutagenesis (Cosmogenetech, Korea) was used to delete the FOXO1 and HFH-3 transcription factor binding sites from the OF-ERV sequence. These transcription factors share the binding site sequence AAACA, which was mutagenized to AGGCA. For this, Cosmogenetech designed the forward 5'-GAT TTT TCA AAC GTA GGC AAA CAA CAG AAA AAT C-3' and reverse 5'-GAT TTT TCT GTT GTT TGC CTA CGT TTG AAA AAT C-3' primers, which were used for PCR amplification with Taq PCR Mastermix (Cosmogenetech, Korea). The total reaction volume was $50 \mu \mathrm{L}$, containing $0.5 \mu \mathrm{L}$ of polymerase, $5 \mu \mathrm{L}$ of dNTPs (2.5 mM each), $10 \mu \mathrm{L}$ of $5 \mathrm{X}$ buffer, $23.5 \mu \mathrm{L}$ of $\mathrm{ddH}_{2} \mathrm{O}, 5 \mu \mathrm{L}$ of each primer $(10 \mathrm{pmol})$ and $1 \mu \mathrm{L}$ of template DNA. The initial denaturation step was held at $95^{\circ} \mathrm{C}$ for $5 \mathrm{~min}$, prior to amplification for $20 \mathrm{cycles}$ at $95^{\circ} \mathrm{C}$ for $1 \mathrm{~min}, 55^{\circ} \mathrm{C}$ for $1 \mathrm{~min}$ and $72^{\circ} \mathrm{C}$ for $4 \mathrm{~min}$, followed by a final elongation step at $72^{\circ} \mathrm{C}$ for $7 \mathrm{~min}$. After amplification, samples were digested with DpnI (New England Biolabs, Beverly, MA) in order to simplify the extraction of only the newly amplified sequences from the PCR products. The digestion reaction was incubated at $37^{\circ} \mathrm{C}$ for 3 hours with a total volume of $35 \mu \mathrm{L}$, which contained $30.5 \mu \mathrm{L}$ of PCR products, $1 \mu \mathrm{L}$ of DpnI and $3.5 \mu \mathrm{L}$ of $10 \mathrm{X}$ buffer. Isolated products were subsequently used for cell transformation.

Cell culture and luciferase assay of OF-ERV9-LTR. SW620 cells, derived from colorectal adenocarcinoma, were grown at $37^{\circ} \mathrm{C}$ in a $5 \%(\mathrm{v} / \mathrm{v}) \mathrm{CO}_{2}$ incubator in Rosewell Park Memorial Institute (RPMI) media (Gibco) supplemented with $10 \%(\mathrm{v} / \mathrm{v})$ heat-inactivated foetal bovine serum (FBS) (Gibco) and 1\% (v/v) antibiotic-antimycotic solution (Gibco, USA). Cells were grown to $70-80 \%$ confluency in 24 -well plates before they were transferred into the relevant experimental medium, supplemented with only $10 \%(\mathrm{v} / \mathrm{v})$ heat-inactivated FBS.

Transfection mixtures included $500 \mathrm{ng}$ of the pGL-4.23 vector (Promega, USA) or a related construct containing either the OF-ERV-LTR sequences from chromosome 9, LTR sequence from chromosome 9 without the transcription binding site for Sox-5, LTR sequence from chromosome 9 without the transcription factor binding sites for Sox-5, GATA-1 and HNF-6, or a mutant form of the LTR sequence from chromosome 9 without any transcription binding sites. Transfection was performed using Lipofectamine 2000 (Invitrogen) as described in 
the manufacturer's protocol. In addition, $100 \mathrm{ng}$ of the pRL-TK plasmid (Promega, USA) was used as a control to normalize the transfection efficiency. Cells were lysed using a $1 \mathrm{X}$ buffer (Promega, USA) as provided and described in the manufacturer's protocol, 24 hours after transfection and then stored at $-80^{\circ} \mathrm{C}$ for a minimum of 3 hours. Firefly and Renilla luciferase activities were assessed using the Dual-Luciferase ${ }^{\circledR}$ Reporter Assay System according to the manufacturer's instructions.

Relative expression analysis of $m k \ln 1$ and OF-miRNA-307 by qPCR amplification. Quantitative polymerase chain reaction (qPCR) primers for $m k \ln 1$ were designed using Primer $3^{52,53}$ as follows: forward, 5'-AGC ATC CAA ACA GCA CAG C-3' and reverse, 5'-CTC CGC CGA GTT AAA TAT CG-3'. The amplification protocol was performed as follows: initial denaturation for $15 \mathrm{~min}$ at $95^{\circ} \mathrm{C} ; 45 \mathrm{cycles}$ of $59^{\circ} \mathrm{C}$ for $15 \mathrm{sec}$ and $72^{\circ} \mathrm{C}$ for $15 \mathrm{sec}$. Furthermore, a standard melting curve ramp ranging from $55^{\circ} \mathrm{C}$ to $99^{\circ} \mathrm{C}$ with a $1^{\circ} \mathrm{C}$ rise on each step was performed. Universal GAPDH primers were used as a reference.

For OF-miRNA-307, each RNA sample was prepared in a reaction volume of $20 \mu \mathrm{L}$. The HB miR Multi Assay KitTM System I (HeimBiotek, Korea) was used for miRNA analysis in a 2-step process. For the initial cDNA synthesis, the HB_I Reverse Transcription (RT) Reaction Kit and its reagents were used according to manufacturer's instructions. Reverse transcription polymerase chain reaction (RT-PCR) was then performed in a thermal cycler (Eppendorf, Hamburg, Germany), using the following conditions: $37^{\circ} \mathrm{C}$ for $60 \mathrm{~min}$ (Step 1) followed by incubation at $95^{\circ} \mathrm{C}$ for $5 \mathrm{~min}$ (Step 2) for one cycle and then held at $4{ }^{\circ} \mathrm{C}$. The final product was stored at $-20^{\circ} \mathrm{C}$ until further use. For the second step, the HB_I Real-time PCR Master mix kit from the HB miR Multi Assay $\mathrm{Kit}^{\mathrm{TM}}$ System I was used according to the manufacturer's suggestions using the Rotor-Gene Q system (QIAGEN, Hilden, Germany). The amplification protocol was performed as follows: initial denaturation for $15 \mathrm{~min}$ at $95^{\circ} \mathrm{C}$; 45 cycles of $95^{\circ} \mathrm{C}$ for $10 \mathrm{sec}$ and $60^{\circ} \mathrm{C}$ for $40 \mathrm{sec}$, and a standard melting curve ramp ranging from $55^{\circ} \mathrm{C}$ to $99^{\circ} \mathrm{C}$ with a $1{ }^{\circ} \mathrm{C}$ rise on each step. Micro RNA U6 was used as a reference. The results were analysed as the relative expression ratio of the target miR-307 (5'- ACA AAA CAC UUU UGG AGU UUC A - $3^{\prime}$ ) to miRNA U6 using the comparative threshold method $(2-\Delta \Delta \mathrm{Ct})$. All experiments were performed in triplicate and the mean values of the resulting relative expression ratios were used for analyses and the generation of charts.

Co-transfection of psi-CHECK-2-mkln1 3'UTR and OF-miRNA-307 mimics. The schematic structure of $m k \ln 1$ shows that it encodes Muskelin N (yellow), while the Kelch3 (red) and Kelch1 (blue) proteins are included in the ORF of $m k \ln 1$. Using the PCR primers forward: $5^{\prime}$-CGC CGG AAT TCT CGA GTC ACC ACT ACA TCC GTG GAG-3' and reverse: 5'-ATT GGA GCT CGA GCT CCC ACA GGA CAG TATG GAAG C-3', the $3^{\prime}$-UTR region of the $m k \ln 1$ gene in olive flounder was amplified and then subsequently cloned into the XhoI cloning site of the psi-CHECK-2 vector (Promega). A forward primer targeting the psi-CHECK-2 vector and reverse primer targeting the $m k \ln 1$ gene were used to verify the orientation of the insert.

HT-29 cells were seeded in a 24 -well dish at $4 \times 10^{4}$ cells/well and grown to $60 \%$ confluency. After 24 hours, transfection was performed with Lipofectamine ${ }^{\mathrm{TM}} 2000$ transfection reagent. OF-miRNA-307 miRNA mimics (Bioneer, Korea) were synthesized and then transfected into the HT-29 cell lines. Negative control miRNA was also included (Bioneer, Korea). The psi-CHECK-2-mkln1-3'UTR vector (100 ng) and miRNA mimics $(0,100$, or $200 \mu \mathrm{M}$ ) were mixed with $50 \mu \mathrm{L}$ of serum-free Opti-MEM (Gibco, USA). Then, $2 \mu \mathrm{L}$ of Lipofectamine ${ }^{\mathrm{TM}} 2000$ were added into each tube and mixed by tapping the tubes. The tubes were incubated at room temperature for $20 \mathrm{~min}$. The mixture was then added to each well, following which the 24 -well plate was rocked gently for $15 \mathrm{sec}$. The plate was then incubated for 24 hours in a $5 \%(\mathrm{v} / \mathrm{v}) \mathrm{CO}_{2}$ humidified atmosphere at $37^{\circ} \mathrm{C}$. Firefly and Renilla luciferase activities were assessed using the Dual-Luciferase ${ }^{\circledR}$ Reporter Assay System according to the manufacturer's instructions.

\section{Data Availability}

The authors declare that all data supporting the findings of this study are available within the article or from the corresponding authors upon reasonable request.

\section{References}

1. Kitano, T., Takamune, K., Kobayashi, T., Nagahama, Y. \& Abe, S. I. Suppression of P450 aromatase gene expression in sex-reversed males produced by rearing genetically female larvae at a high water temperature during a period of sex differentiation in the Japanese flounder (Paralichthys olivaceus). J. Mol. Endocrinol. 23, 167-176 (1999).

2. Yoshinaga, N. et al. Sexually dimorphic expression of a teleost homologue of Müllerian inhibiting substance during gonadal sex differentiation in Japanese flounder, Paralichthys olivaceus. Biochem. Biophys. Res. Commun. 322, 508-513 (2004).

3. Fan, Z. et al. Gonadal transcriptome analysis of male and female olive flounder (Paralichthys olivaceus). Biomed. Res. Int. 291067, 10 (2014).

4. Huang, L. et al. De Novo assembly of the Japanese flounder (Paralichthys olivaceus) spleen transcriptome to identify putative genes involved in immunity. PLoS One 10, e0117642 (2015).

5. Isshiki, I., Nishizawa, T., Kobayashi, T., Nagano, T. \& Miyazaki, T. An outbreak of VHSV (viral haem-orrhagic septicemia virus) infection in farmed Japanese flounder Paralichthys olivaceus in Japan. Dis. Aquat. Organ 47, 87-99 (2001).

6. Moustafa, E., Naota, M., Morita, T., Tange, N. \& Shimada, A. Pathological Study on the Scuticociliatosis Affecting Farmed Japanese Flounder (Paralichthys olivaceus) in Japan. Journal of Veterinary Medical Science 72, 1359-1362 (2010).

7. Simora, R. M. et al. Molecular cloning and antiviral activity of IFN-b promoter stimulator-1 (IPS-1) gene in Japanese flounder, Paralichthys olivaceus. Fish Shellfish Immunol. 29, 979-986 (2010).

8. Wang, W. et al. Detection of alternative splice and gene duplication by RNA sequencing in Japanese flounder Paralichthys olivaceus. G3 (Bethesda) 4, 2419-2424 (2014).

9. Lee, H. E., Ayarpadikannan, S. \& Kim, H. S. Role of Transposable elements in genomics rearrangement, evolution, gene regulation and epigenetics in primates. Genes \& Genetic Systems 90(5), 245-257 (2015).

10. Kim, H. S. Genomic impact, chromosomal distribution and transcriptional regulation of HERV elements. Mol. Cells 33, 539-544 (2012).

11. Gonzalez-Cao, M. et al. Human endogenous retroviruses and cancer. Cancer Biol. Med. 13(4), 483-488 (2016). 
12. Johanning, G. L. et al. Expression of human endogenous retrovirus- $\mathrm{K}$ is strongly associated with the basal-like breast cancer phenotype. Sci. Rep. 7, 41960 (2017).

13. Akopov, S. B., Nikolaev, L. G., Khil, P. P., Lebedev, Y. B. \& Sverdlov, E. D. Long terminal repeats of human endogenous retrovirus K family (HERV-K) specifically bind host cell nuclear proteins. FEBS Letters 421(3), 1873-3468 (1998).

14. Vinogradova, T. V. et al. Solitary Human Endogenous Retroviruses-K LTRs Retain Transcriptional Activity in Vivo, the mode of Which Is Different in Different Cell Types. Virology 290(1), 83-90 (2001).

15. Hu, T. et al. Long non-coding RNAs transcribed by ERV-9 LTR retrotransposon act in cis to modulate long-range LTR enhancer function. Nucleic Acids Research 45(8), 4479-4492 (2017).

16. Thompson, P. J., Macfarlan, T. S. \& Lorincz, M. C. Long terminal repeats: From parasitic elements to building blocks of the transcriptional regulatory repertoire. Molecular Cell 62, 766-776 (2016).

17. Ruda, V. M. et al. Tissue Specificity of enhancer and promoter activities of a HERV-K(HML-2) LTR. Virus Research 104(1), 11-16 (2004).

18. Choung, E. B., Rumi, M. A. K., Soares, M. J. \& Baker, J. C. Endogenous retroviruses function as species-specific enhancer elements in the placenta. Nature Genetics 45(3), 325-329 (2013)

19. Nam, G. H. et al. Expression and promoter activity of endogenous retroviruses in the Olive flounder (Paralichthys olivaceus). Genes Genom. 38, 539-545 (2016).

20. Bartel, D. P. MicroRNAs: genomics, Biogenesis, mechanisms, and Function. Cell 116, 281-297 (2004).

21. Friedman, R. C., Farh, K. K.-H., Burge, C. B. \& Bartel, D. P. Most mammalian mRNAs are conserved targets of microRNAs. Genome Res. 19, 92-105 (2008).

22. Ambros, V. et al. A uniform system for microRNA annotation. RNA 9, 277-279 (2003).

23. Piriyapongsa, J. \& Jordan, I. K. A family of human microRNA genes from miniature inverted-repeat transposable elements. PLOS ONE 2(2), e203 (2007).

24. Piriyapongsa, J., Mariño-Ramírez, L. \& Jordan, I. K. Origin and evolution of human microRNAs from transposable elements. Genetics 176, 1323-1337 (2007).

25. Crooks, G. E., Hon, G., Chandonia, J. M. \& Brenner, S. E. WebLogo: A sequence logo generator. Genome Research 14, 1188-1190 (2004).

26. Schneider, T. D. \& Stephens, R. M. Sequence Logos: A new way to display consensus sequences. Nucleic Acids Res. 18, 6097-6100 (1990).

27. Ashburner, M. et al. Gene Ontology: tool for the unification of biology. Nature Genet. 25(1), 25-29 (2000).

28. Huh, J. W. et al. Cooperative exonization of MaLR and AluJo elements contributed an alternative promoter and novel spliced variants of RNF19. Gene 424, 63-70 (2008).

29. Medstrand, P., Landry, J. R. \& Mager, D. L. Long terminal repeats are used as alternative promoters of the endothelin B receptor and apolopoprotein CI genes in humans. J. Biol. Chem. 276, 1896-1903 (2001).

30. Wang, T. et al. Species-specific endogenous retroviruses shape the transcriptional network of the human tumor suppressor protein p53. Proc. Natl. Acad. Sci. USA 104(47), 18613-18618 (2007).

31. Bourque, G. et al. Evolution of the mammalian transcription factor binding repertoire via transposable elements. Genome Res. 18(11), 1752-1762 (2008)

32. Sundaram, V. et al. Widespread contribution of transposable elements to the innovation of gene regulatory networks. Genome Res. 24(12), 1963-1976 (2014)

33. Jung, Y. D. et al. Activity analysis of LTR12C as an effective regulatory element of the RAE1 gene. Gene 634, 22-28 (2017).

34. Wunderle, V. M., Critcher, R., Ashworth, A. \& Goodfellow, P. N. Cloning and Characterization of SOX5, a New Member of the Human SOX Gene Family. Genomics 36(2), 354-358 (1996).

35. Lefebvre, V. The SoxD transcription factors - Sox5, Sox6, and Sox13 - are key cell fate modulators. Int. J. Biochem. Cell Biol. 42(3), 429-432 (2010).

36. Liu, C. F. \& Lefebvre, V. The transcription factors SOX9 and SOX5/SOX6 cooperate genome-wide through super-enhancers to drive chondrogenesis. Nucleic Acids Research 43(17), 8183-8203 (2015).

37. Smits, P. et al. The Transcription Factors L-Sox5 and Sox6 Are Essential for Cartilage Formation. Developmental Cell 1(1), 277-290 (2001).

38. Kamachi, Y. \& Kondoh, H. Sox proteins: regulators of cell fate specification and differentiation. Development 140, 4129-4144 (2013).

39. Mata-Rocha, M. et al. The transcription factors Sox 5 and Sox 9 regulate Catsper 1 gene expression. FEBS Letters 588(18), 3352-3360 (2014).

40. Cheng, J. H., Pan, D. Z. C., Tsai, Z. T. Y. \& Tsai, H. K. Genome-wide analysis of enhancer RNA in gene regulation across 12 mouse tissues. Scientific reports 5, 12648 (2015).

41. Smalheiser, N. R. \& Torvik, V. I. Mammalian microRNAs derived from genomic repeats. TRENDS in Genetics 21(6), 322-326 (2005).

42. Jo, A., Lee, H. E. \& Kim, H. S. Identification and expression analysis of a novel miRNA derived from ERV-E1 LTR in Equus caballus. Gene 687, 238-245 (2019).

43. Suzuki, H. I., Young, R. A. \& Sharp, P. A. Super Enhancer-Mediated RNA Processing Revealed by Integrative MicroRNA Network Analysis. Cell 168(6), 1000-1014 (2017).

44. Xiao, M. et al. MicroRNAs activate gene transcription epigenetically as an enhancer trigger. RNA Biology 14(10), 1326-1334 (2017),

45. Sperber, G., Lövgren, A., Eriksson, N., Benachenhou, F. \& Blomberg, J. RetroTector online, a rational tool for analysis of retroviral elements in small and medium size vertebrate genomic sequences. BMC Bioinformatics 10, S4 (2009).

46. Hall, T. A. BioEdit: a user-friendly biological sequence alignment editor and analysis program for Windows 95/98/NT. Nucleic Acids Symposium Series 41, 95-98 (1999).

47. Kumar, S., Stecher, G. \& Tamura, K. MEGA7: Molecular Evolutionary Genetics Analysis version 7.0 for bigger datasets. Molecular Biology and Evolution 33, 1870-1874 (2016).

48. Rehmsmeier, M., Steffen, P., Hochsmann, M. \& Giegerich, R. Fast and effective prediction of microRNA/target duplexes. RNA 10, $1507-1517$ (2004).

49. Kim, D., Park, E. \& Kim, J. Karyotype of nine species of the Korean catfishes (Teleostomi:Siluriformes). Korean J. Genet. 4, 57-68 (1982).

50. Waminal, N. E. \& Kim, H. H. Dual-color FISH karyotype and rDNA distribution analyses on four Cucurbitaceae species. Horticulture, Environment, and Biotechnology 53(1), 49-56 (2012).

51. Matoba, H., Mizutani, T., Nagano, K., Hoshi, Y. \& Uchiyama, H. Chromosomal study of lettuce and its allied species (Lactuca spp., Asteraceae) by means of karyotype analysis and fluorescence in situ hybridization. Hereditas 144, 235-243 (2007).

52. Untergasser, A. et al. Primer3 - new capabilities and interfaces. Nucleic Acids Research 40(15), el15 (2012).

53. Koressaar, T. \& Remm, M. Enhancements and modifications of primer design program Primer3. Bioinformatics 23(10), 1289-1291 (2007). 


\section{Acknowledgements}

This research was supported by the Marine Biotechnology Program of the Korea Institute of Marine Science and Technology Promotion (KIMST), funded by the Ministry of Oceans and Fisheries (MOF) (No. 20140428). Additionally, this work was supported by a grant from the National Institute of Fisheries Science (R2018001).

\section{Author Contributions}

H.E.L prepared and revised the manuscript. H.E.L., A.J., J.I. and H.H.K. performed the experiments. H.J.C., W.J.K., D.S.K., W.K., Y.T.J. and H.S.K. commented on the manuscript.

\section{Additional Information}

Supplementary information accompanies this paper at https://doi.org/10.1038/s41598-019-50492-7.

Competing Interests: The authors declare no competing interests.

Publisher's note Springer Nature remains neutral with regard to jurisdictional claims in published maps and institutional affiliations.

(c) (1) Open Access This article is licensed under a Creative Commons Attribution 4.0 International License, which permits use, sharing, adaptation, distribution and reproduction in any medium or format, as long as you give appropriate credit to the original author(s) and the source, provide a link to the Creative Commons license, and indicate if changes were made. The images or other third party material in this article are included in the article's Creative Commons license, unless indicated otherwise in a credit line to the material. If material is not included in the article's Creative Commons license and your intended use is not permitted by statutory regulation or exceeds the permitted use, you will need to obtain permission directly from the copyright holder. To view a copy of this license, visit http://creativecommons.org/licenses/by/4.0/.

(C) The Author(s) 2019 